TRANSACTIONS OF THE

AMERICAN MATHEMATICAL SOCIETY

Volume 362, Number 9, September 2010, Pages 4569-4580

S 0002-9947(10)05113-5

Article electronically published on April 14, 2010

\title{
FLOWS WITHOUT WANDERING POINTS ON COMPACT CONNECTED SURFACES
}

\author{
MILTON COBO, CARLOS GUTIERREZ, AND JAUME LLIBRE
}

\begin{abstract}
Given a compact 2-dimensional manifold $M$ we classify all continuous flows $\varphi$ without wandering points on $M$. This classification is performed by finding finitely many pairwise disjoint open $\varphi$-invariant subsets $\left\{U_{1}, U_{2}, \ldots, U_{n}\right\}$ of $M$ such that $\bigcup_{i=1}^{n} \overline{U_{i}}=M$ and each $U_{i}$ is either a suspension of an interval exchange transformation, or a maximal open cylinder made up of closed trajectories of $\varphi$.
\end{abstract}

\section{IntRoduCtion AND STATEMENT OF THE MAIN RESUlts}

Let $M$ be a $C^{\infty}$ compact connected 2-dimensional manifold without boundary. Let $\varphi: \mathbb{R} \times M \rightarrow M$ be a flow (on $M$ ); that is, $\varphi$ is continuous and

(i) $\varphi(0, p)=p$ for all $p \in M$;

(ii) $\varphi(t, \varphi(s, p))=\varphi(t+s, p)$ for all $p \in M$ and all $t, s \in \mathbb{R}$.

We say that the flow $\varphi: \mathbb{R} \times M \rightarrow M$ is without wandering points if for every pair $(\mathcal{V}, R)$ consisting of an open subset $\mathcal{V}$ of $M$ and a real number $R>0$, there exists a real number $|t|>R$ such that $\varphi_{t}(\mathcal{V}) \cap \mathcal{V} \neq \emptyset$, where $\varphi_{t}(\mathcal{V}):=\{\varphi(t, p): p \in \mathcal{V}\}$. Continuous area-preserving flows are examples of flows without wandering points.

The theorem below is our main result on the dynamics of continuous flows on compact connected surfaces. To state it we shall need the following definitions.

We define the $\omega$-limit set (resp. $\alpha$-limit set) of $p \in M$ as the set $\omega(p)$ (resp. $\alpha(p)$ ) made up of the $q \in M$ such that for some sequence of real numbers $t_{n} \rightarrow \infty$ $\left(\right.$ resp. $\left.t_{n} \rightarrow-\infty\right), \varphi\left(t_{n}, p\right) \rightarrow q$.

The positive (resp. negative) half-trajectory or semi-trajectory $\gamma_{p}^{+}$(resp. $\gamma_{p}^{-}$) through the point $p \in M$ is the set $\{\varphi(t, p): t \geq 0\}$ (resp. $\{\varphi(t, p): t \leq 0\}$ ). The orbit or trajectory $\gamma_{p}$ through the point $p \in M$ is the set $\gamma_{p}^{+} \cup \gamma_{p}^{-}$. The $\omega$-limit set (respectively $\alpha$-limit set) of an orbit $\gamma$ is the set $\omega(p)$ (respectively $\alpha(p)$ ) for some $p \in \gamma$.

A point $p$ is a fixed point of $\varphi$ if the orbit of $p$ is the set $\{p\}$. An orbit is said to be regular if it is not reduced to a fixed point.

Received by the editors May 10, 2008.

2000 Mathematics Subject Classification. Primary 37B05, 37B10, 47B36, $47 \mathrm{~B} 37$.

Key words and phrases. Interval exchange transformation, flows in compact surfaces, wandering sets.

Unfortunately the second author died during the period that this manuscript was submitted.

(C)2010 American Mathematical Society 
An orbit is periodic or closed if it is homeomorphic to a circle. A trajectory $\gamma$ which is neither a fixed point nor a closed trajectory is said to be nontrivial recurrent if either $\gamma \subset \alpha(\gamma)$ or $\gamma \subset \omega(\gamma)$.

Recall that a fixed point $p$ of the flow $\varphi$ is a center if there is a neighborhood of $p$ where all orbits, excepting the fixed point, are periodic.

We say that a fixed point of $\varphi$ is a multi-saddle if it has a neighborhood which splits into a finite union of an even number of $n \geq 2$ hyperbolic sectors; see Figure 1 for the cases $n=2$ and $n=8$.

A regular trajectory $\gamma$ such that $\alpha(\gamma)$ and $\omega(\gamma)$ are multi-saddle points is said to be a multi-saddle connection; the possibility $\alpha(\gamma)=\omega(\gamma)$ is not excluded.

A closed connected subset $\Gamma$ of $M$ made up of (finitely many) multi-saddle points and multi-saddle connections will be said to be a graph. A graph $\Gamma$ is said to be an attracting one-circuit (resp. a repelling one-circuit) if there exists an annulus $A$ such that $A \cap \Gamma=\emptyset, \Gamma \subset \bar{A}$, and for all $p \in A, \omega(p)=\Gamma($ resp. $\alpha(p)=\Gamma)$. Let $\gamma$ be

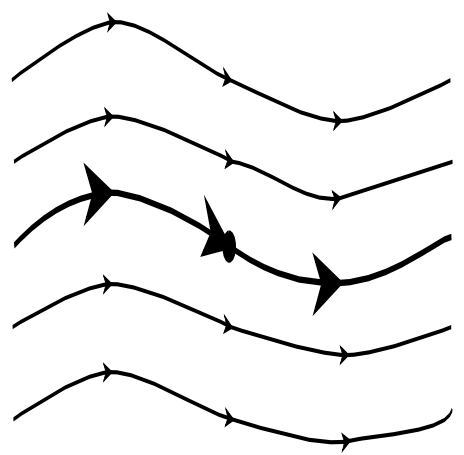

(a)

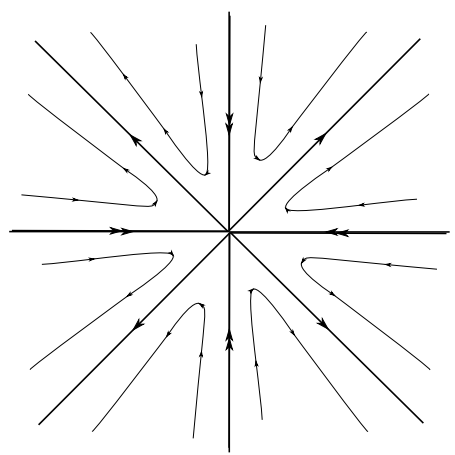

(b)

Figure 1. Multi-saddles with $n=2$ and $n=8$ hyperbolic sectors.

a periodic orbit of $\varphi$. We say that $\gamma$ is two-sided if it has a neighborhood which is homeomorphic to a cylinder. Otherwise we say that $\gamma$ is one-sided, in which case $\gamma$ possesses a neighborhood homeomorphic to a Möbius band.

We say that a $\varphi$-invariant submanifold $\widetilde{C}$ of $M$ is a semi-cylindric region if the following conditions are satisfied: either $\widetilde{C}=M$ is a 2-dimensional torus (or a simple 2-torus) filled out by two-sided closed orbits, or

(i) it contains a $\varphi$-invariant open cylinder $C$ filled out by closed orbits of $\varphi$ (i.e. the flow $\varphi$ restricted to $C$ is topologically equivalent to the constant vector field $(1,0)$ defined on $\mathbb{R} / \mathbb{Z} \times(-1,1))$;

(ii) $\widetilde{C}=C \cup \partial C$;

(iii) when $\partial C$ has two connected components, each of them is either a center point, or a one-sided closed orbit, or else a graph of $\varphi$; and

(iv) when $\partial C$ has one connected component, it is a graph of $\varphi$.

Some elementary properties of the semi-cylindrical regions are collected in what follows.

Remark 1. Suppose that $\widetilde{C}=C \cup \partial C$ is a semi-cylindrical region. Then the following statements hold. 
(a) If $\partial C$ consists of two center points $p$ and $q$, then $M=C \cup\{p\} \cup\{q\}$ (having Euler characteristic 2) is the 2 -sphere.

(b) If $\partial C$ consists of a center point $p$ and a one-sided closed orbit $\gamma$, then $M=C \cup\{p\} \cup \gamma$ (having Euler characteristic 1) is the 2-projective space.

(c) If $\partial C$ consists of two one-sided closed orbits $\gamma, \sigma$, then $M=C \cup \sigma \cup \gamma$ is the Klein bottle.

A quasi-minimal set of $\varphi$ is the closure of a nontrivial recurrent trajectory. A compact connected subset $Q$ of $M$ will be said to be a semi-submanifold of $M$ if there exist a compact 2-dimensional manifold $S$ (with or without boundary), a finite or empty subset $F$ of $\partial S$ (the boundary of $S$ ) and a partition $\left\{F_{1}, F_{2}, \ldots, F_{\ell}\right\}$ of $F$ such that if $\sim$ denotes the equivalence relation in $S$ defined by $a \sim b$ if and only if either $a=b$ or $\{a, b\} \subset F_{i}$, for some $i \in\{1,2, \ldots, k\}$, then $Q$ is homeomorphic to the quotient space $S / \sim$. See Figure 2 .

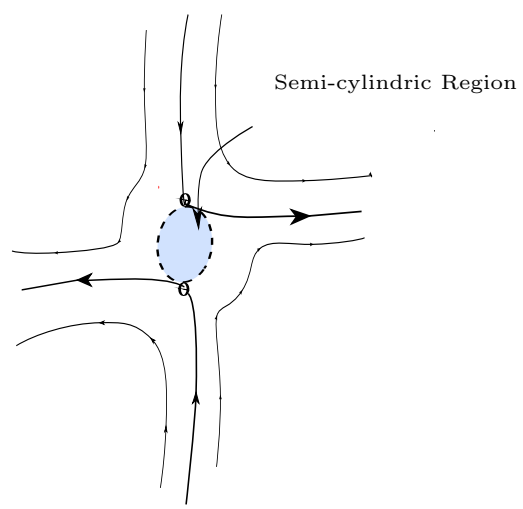

(a)

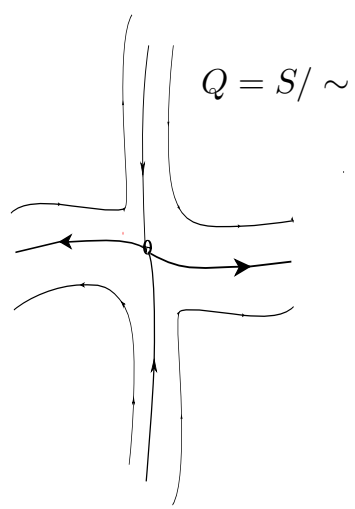

(b)

Figure 2. $S=\mathbb{R}^{2} \backslash\left\{(x, y): x^{2}+y^{2} \leq 1, x \neq 0\right\}$.

A $\varphi$-invariant semi-submanifold $Q$ of $M$ with or without boundary is a quasiminimal recurrence region of $\varphi$ if

(i) every orbit in the interior of $Q$, with the possible exception of finitely many ones, is dense in $Q$ (and so nontrivial recurrent);

(ii) when an orbit in the interior of $Q$ is not dense, then it is either a multisaddle point, or a multi-saddle connection;

(iii) when $\partial Q$ is not empty, it is made up of finitely many graphs of $\varphi$.

Remark 2. Let $Q$ be a quasi-minimal recurrence region. If $\partial Q$ is empty and $\varphi$ has no multi-saddles in $Q$ of negative Poincaré-Hopf index, then $Q=M$ is the 2 -torus. In fact, as $M$ must have Euler characteristic 0 and the Klein bottle does not support nontrivial recurrent trajectories [8] (see also [2]) $M$ must be the 2-torus.

Our main result is the following one.

Theorem 3. Let $M$ be a boundaryless compact connected smooth surface and let $\varphi: \mathbb{R} \times M \rightarrow M$ be a continuous flow without wandering points and having finitely many fixed points. Then the following statements hold. 
(a) Every fixed point of $\varphi$ is either a center or a multi-saddle.

(b) The manifold $M$ is decomposed into the union of finitely many $\varphi$-invariant semi-submanifolds, having pairwise disjoint interiors, and being of one of the following types: semi-cylindric or quasi-minimal recurrence regions.

This paper is organized as follows. In section 2 we characterize the local phase portraits at isolated fixed points; in that section we also consider the structure of the limit sets of trajectories. In section 3 we define interval exchange transformations and their suspensions to compact surfaces. Section 4 is devoted to completing the proofs of Theorems 3 and 12. This last theorem gives a precise description of the structure of the quasi-minimal recurrence regions.

\section{Fixed POINTS AND LIMIT SETS}

The next result classifies the local phase portraits of the fixed points of a flow $f$ satisfying the assumptions of Theorem 3 .

Lemma 4. In the assumptions of Theorem 3, every fixed point of $\varphi$ is either a center or a multi-saddle.

Proof. Let $p \in M$ be a fixed point of $\varphi$ different from a center. By the Local Sector Classification Theorem (see [1, pp. 313-314], [5, Chapter VII, page 161]), $p$ has arbitrarily small canonical neighborhoods homeomorphic to compact discs whose boundaries are circles having the least possible number of tangencies with the foliation $\mathcal{F}$. Any such neighborhood $D$ containing $p$ in its interior splits into a union of finitely many hyperbolic, parabolic and elliptic sectors. As $\varphi$ has no wandering points, $D$ contains neither elliptic sectors nor parabolic sectors. This implies that $p$ has to be a multi-saddle point.

We will need the following generalization of the classical Poincaré-Bendixson theorem (see for example [9]).

Theorem 5. Let $\varphi: \mathbb{R} \times M \rightarrow M$ be a continuous flow with finitely many fixed points on a compact surface $M$ and let $l$ be a positive (negative) semi-trajectory of $\varphi$. Then the $\omega$-limit ( $\alpha$-limit) of $l$ is of one of the following types:

(i) a fixed point,

(ii) a periodic trajectory,

(iii) an attracting (resp. a repelling) one-circuit, or

(iv) a quasi-minimal set.

Corollary 6. Let $\varphi: \mathbb{R} \times M \rightarrow M$ be as in Theorem 5 and suppose that $\varphi$ is without wandering points. If $\gamma$ is a regular trajectory of $\varphi$, then one of the following alternatives occurs:

(i) $\gamma$ is a multi-saddle connection;

(ii) $\gamma$ is a periodic trajectory;

(iii) $\gamma$ is a nontrivial recurrent trajectory.

Proof. If $\varphi$ has no wandering points, one-circuits cannot be the $\omega$-limit or $\alpha$-limit of any trajectory; i.e., one-circuits cannot exist. By the same reason a periodic trajectory $l$ cannot be the $\omega$-limit or $\alpha$-limit of any trajectory other than $l$. Therefore if $l$ is not a periodic trajectory, by Lemma 4 and Theorem 5, its $\omega$-limit and $\alpha$-limit sets can only be multi-saddle points or quasi-minimal sets. 
When $\varphi$ is without wandering points and $\gamma$ is a two-sided periodic orbit, it has open neighborhoods which are cylinders filled out by two-sided closed orbits. The union $V$ of all these cylindrical neighborhoods is a semi-cylindric region; the case in which $V=M$ is a 2 -torus is not excluded.

\section{Suspension of a minimal CET}

We denote by $\mathbb{S}^{1}$ the unit circle of $\mathbb{R}^{2}$. A circle exchange transformation, or simply a CET, will be a $C^{1}$ injective map $E$ on the circle $\mathbb{S}^{1}$ and whose domain of definition $\operatorname{Dom}(E)$ is the complement in $\mathbb{S}^{1}$ of a finite or empty subset $\mathcal{N}(E)$, and such that for all $x \in \mathbb{S}^{1} \backslash \mathcal{N}(E)$, the derivative $E^{\prime}(x)$ satisfies $\left|E^{\prime}(x)\right|=1$. By abuse of notation we will sometimes write $E: \mathbb{S}^{1} \rightarrow \mathbb{S}^{1}$ as if $E$ were defined everywhere.

Traditionally a generalized interval exchange transformation is defined as an injective map $T:[0,1) \rightarrow[0,1)$ such that, for some partition $0=s_{1}<s_{2}<\cdots<$ $s_{m}<s_{m+1}=1$ of $[0,1)$ and for some numbers $d_{1}, \ldots, d_{m}$, we have

$$
T(x)= \pm x+d_{j}, \quad \text { for } x \in\left[s_{j}, s_{j+1}\right) \text { and } 1 \leq j \leq m .
$$

If $T$ preserves orientation (i.e., $T^{\prime}(x) \equiv 1$ ) it is called simply an interval exchange map, but if $T^{\prime}(x)=-1$ in some interval of continuity of $T$, then we say that $T$ has flips.

If we identify $\mathbb{S}^{1}$ with the interval $[0,1)$, the map $T$ induces a map $\tilde{T}: \mathbb{S}^{1} \rightarrow \mathbb{R} / \mathbb{Z}$ which is similar, but not equal, to a CET because $\tilde{T}$ would be defined everywhere in $\mathbb{S}^{1}$ while in general a CET is not defined in all $\mathbb{S}^{1}$.

Let $E$ be a CET and $a \in \mathcal{N}(E)$. Then the lateral limits

$$
E^{+}(a):=\lim _{x \rightarrow a^{+}} E(x) \text { and } E^{-}(a):=\lim _{x \rightarrow a^{-}} E(x)
$$

always exist. Observe that, under the given conditions, the inverse map $E^{-1}$ of $E$ is well defined and is also a CET. An orbit of $E$ is a maximal set of the form

$$
\ldots, E^{-i}(x), \ldots, E^{-1}(x), x, E(x), \ldots, E^{j}(x), \ldots,
$$

where $x$ is a point of $\mathbb{S}^{1}$ and $i, j \in \mathbb{N} \cup\{0\}$. So, any finite orbit of $E$ of length $n \in \mathbb{N} \cup\{0\}$ is a finite sequence of the form

$$
x, E(x), E^{2}(x), \ldots, E^{n-1}(x),
$$

whose elements are pairwise different and which satisfies one of the following two alternatives:

(i) $E^{n}(x)=x$, in which case the orbit is called periodic, or

(ii) $x \in \operatorname{Dom}\left(E^{n-1}\right), x \in \mathcal{N}\left(E^{-1}\right)$ and $E^{n-1}(x) \in \mathcal{N}(E)$, in which case the orbit through $x$ will be said to be a connection between singularities.

A short remark is necessary now. It is proved in [7] that an orientation-preserving interval exchange map $T$ with no saddle connections is minimal (i.e., every orbit is dense). On the other hand if $T$ has flips but no saddle connections, then $T^{2}$ is orientation-preserving and also has no saddle connections; therefore $T^{2}$ and $T$ are minimal. Hence the following result easily follows from the first lemma on page 27 of [7:

Lemma 7 (Keane). Let $E: \mathbb{S}^{1} \rightarrow \mathbb{S}^{1}$ be a CET such that $\mathcal{N}(E) \neq \emptyset$. If $E$ has no connection between singularities, then every orbit of $E$ is dense; i.e., $E$ is minimal. 
Given a CET $E: \mathbb{S}^{1} \rightarrow \mathbb{S}^{1}$ we consider the equivalence relation $\simeq$ in $\mathbb{S}^{1} \times[-1,1]$ defined by the relation

$$
(a, 1) \sim(b,-1) \text { if and only if } b \text { is equal to } E^{+}(a) \text { or } E^{-}(a) .
$$

It is easy to check that every equivalence class of $\sim$ always has an even number of elements. If $a \in \mathbb{S}^{1} \backslash \mathcal{N}(E)$, then $\{(a, 1),(E(a),-1)\}$ form a two-point equivalence class. If $a \in \mathcal{N}(E)$, there may be more than two elements in the equivalence class of $(a, 1)$. We denote by $F_{1}, F_{2}, \ldots, F_{\ell}$ all the equivalence classes of $\sim$ possessing some element in $(\mathcal{N}(E) \times\{1\}) \cup\left(\mathcal{N}\left(E^{-1}\right) \times\{-1\}\right)$. Notice that

$$
\bigcup_{i=1}^{\ell} F_{i}=(\mathcal{N}(E) \times\{1\}) \cup\left(\mathcal{N}\left(E^{-1}\right) \times\{-1\}\right) \text {. }
$$

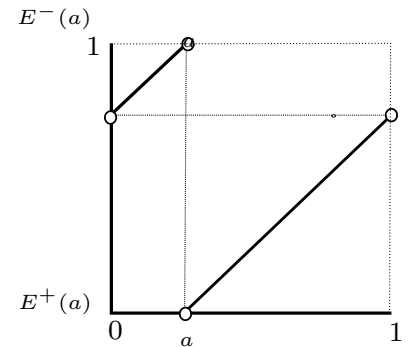

(a)

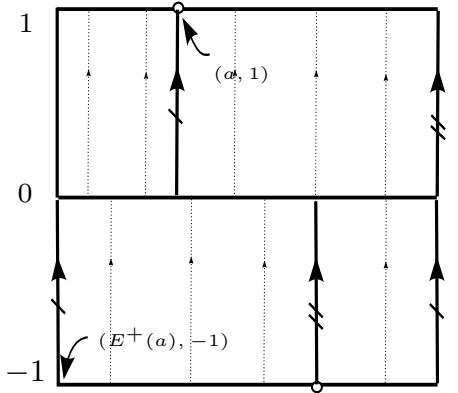

(b)

FigURE 3. Suspension of a rotation.

Let $S(E)$ be the partition of $\mathbb{S}^{1} \times[-1,1]$ whose non-one-point atoms are exactly the equivalence classes of $\sim$.

Lemma 8. Let $E: \mathbb{S}^{1} \rightarrow \mathbb{S}^{1}$ be a CET such that $\mathcal{N}(E) \neq \emptyset$. Let $\Pi: \mathbb{S}^{1} \times[-1,1] \rightarrow$ $S(E)$ be the canonical projection and provide $S(E)$ with the quotient topology. Then $S(E)$ can be given with the structure of a smooth compact connected boundaryless 2-manifold satisfying the following two statements.

(a) The vertical foliation on $\mathbb{S}^{1} \times[-1,1]$ (i.e., the one induced by the vector field $(0,1))$ induces a smooth orientable foliation $\mathcal{F}$ on $S(E)$ whose singularities are the points $\Pi\left(F_{i}\right), i=1, \ldots, \ell$. Each $\Pi\left(F_{i}\right)$ is a multi-saddle point, and the number of separatrices equals the cardinality of the elements of $F_{i}$ : if $(a, 1) \in F_{i} \cap(\mathcal{N}(E) \times\{1\})$ (resp. $\left.(a,-1) \in F_{i} \cap(\mathcal{N}(E) \times\{-1\})\right)$, then $\Pi(\{a\} \times(-1,1))$ is a semi-trajectory contained in a stable (resp. unstable) separatrix of $\Pi\left(F_{i}\right)$.

(b) There exists a smooth flow $\Phi$ in $S(E)$ such that

(i) the fixed points of $\Phi$ are the singularities of $\mathcal{F}$;

(ii) the regular trajectories of $\Phi$ are the regular trajectories of $\mathcal{F}$;

(iii) $\Phi$ is transversal to $\mathbb{S}^{1} \times\{0\}$; and

(iv) the forward Poincaré map $\mathbb{S}^{1} \times\{0\} \rightarrow \mathbb{S}^{1} \times\{0\}$ induced by $\Phi$ is $(x, 0) \mapsto$ $(E(x), 0)$.

The pair $(\Phi, S(E))$ is said to be a suspension of $E$. 
Proof. The usual Euclidean metric on $\mathbb{R} \times[-1,1]$ induces, via the canonical projection,

$$
\mathbb{R} \times[-1,1] \rightarrow \mathbb{S}^{1} \times[-1,1],
$$

a smooth Riemannian structure on $\mathbb{S}^{1} \times[-1,1]$. Let $\rho$ be the corresponding distance function on $\mathbb{S}^{1} \times[-1,1]$. As $E$ is an isometry, the canonical smooth Riemannian structure of $\mathbb{S}^{1} \times[-1,1]$ induces a smooth Riemannian structure on

$$
\Pi\left(\mathbb{S}^{1} \times[-1,1] \backslash\left(\mathcal{N}(E) \times\{1\} \cup \mathcal{N}\left(E^{-1}\right) \times\{-1\}\right)\right),
$$

in such a way that $\Pi$ restricted to

$$
\mathbb{S}^{1} \times[-1,1] \backslash\left(\mathcal{N}(E) \times\{1\} \cup \mathcal{N}\left(E^{-1}\right) \times\{-1\}\right)
$$

is an isometry. This implies that the vertical foliation on $\mathbb{S}^{1} \times[-1,1]$ (i.e., the one induced by the vector field $(0,1)$; see Figure 3$)$ induces a smooth orientable foliation $\mathcal{F}$, without singularities, on $S(E) \backslash\left(\bigcup_{i=1}^{\ell} \Pi\left(F_{i}\right)\right)$.

We prove now that, for all $i=1,2, \ldots, \ell$, every $\Pi\left(F_{i}\right)$ is a multi-saddle point and that $S(E)$ has a smooth structure around $\Pi\left(F_{i}\right)$.

Take $\varepsilon>0$ small so that all the half-discs

$$
D(z)=\left\{p \in \mathbb{S}^{1} \times[-1,1]: \rho(p, z) \leq \varepsilon\right\},
$$

with $z \in \mathcal{N}(E) \times\{1\} \cup \mathcal{N}\left(E^{-1}\right) \times\{-1\}$, are pairwise disjoint. We orient the closed half-circles

$$
C(z)=\left\{p \in \mathbb{S}^{1} \times[-1,1]: \rho(p, z)=\varepsilon\right\},
$$

with $z \in \mathcal{N}(E) \times\{1\} \cup \mathcal{N}\left(E^{-1} \times\{-1\}\right)$, counterclockwise with respect to the point $z$.

We fix $\sigma \in\{1,2, \ldots, \ell\}$ and take $z_{1} \in F_{\sigma}$. Let $x_{1}$ (resp. $y_{1}$ ) be the starting (resp. ending) endpoint of the oriented closed half-circle $C\left(z_{1}\right)$. There exists exactly one point $z_{2} \in \mathcal{N}\left(E^{-1}\right) \times\{-1\}$ such that $z_{1} \sim z_{2}$ (as in equation (11) right after Lemma 7) and if $x_{2}$ (resp. $y_{2}$ ) is the starting (resp. ending) endpoint of $C\left(z_{2}\right)$, then $\Pi\left(y_{1}\right)=\Pi\left(x_{2}\right)$. Similarly, there exists exactly one point $z_{3} \in \mathcal{N}(E) \backslash\left\{z_{1}\right\}$ such that $z_{2} \sim z_{3}$ and if $x_{3}$ (resp. $\left.y_{3}\right)$ is the starting (resp. ending) endpoint of $C\left(z_{3}\right)$, then $\Pi\left(y_{2}\right)=\Pi\left(x_{3}\right)$.

In this way we construct a finite sequence

$$
\left(x_{1}, y_{1}, z_{1}\right),\left(x_{2}, y_{2}, z_{2}\right), \ldots,\left(x_{2 m+1}, y_{2 m+1}, z_{2 m+1}\right)
$$

of an odd number of terms such that

(i) $z_{1}, z_{2}, \ldots, z_{2 m}$ are pairwise different, but $z_{2 m+1}=z_{1}$; also $z_{1}, z_{3}, \ldots, z_{2 m-1}$ $\in \mathcal{N}(E) \times\{1\}$ and $z_{2}, z_{4}, \ldots, z_{2 m} \in \mathcal{N}\left(E^{-1}\right) \times\{-1\}$

(ii) $z_{1} \sim z_{2}, z_{2} \sim z_{3}, \ldots, z_{2 m} \sim z_{2 m+1}=z_{1}$ and so $F_{\sigma}=\left\{z_{1}, z_{2}, \ldots, z_{2 m}\right\}$;

(iii) for every $(a, 1) \in F_{\sigma} \cap(\mathcal{N}(E) \times\{1\})\left(\right.$ resp. $\left.(a,-1) \in F_{\sigma} \cap(\mathcal{N}(E) \times\{-1\})\right)$, $\Pi(\{a\} \times(-1,1))$ is a semi-trajectory contained in a stable (resp. unstable) separatrix of the singularity $\Pi\left(F_{\sigma}\right)$. In this way, $\Pi\left(F_{\sigma}\right)$ is a multi-saddle point of $\mathcal{F}$ with $2 m$ separatrices.

(iv) $\Pi\left(y_{1}\right)=\Pi\left(x_{2}\right), \Pi\left(y_{2}\right)=\Pi\left(x_{3}\right), \ldots, \Pi\left(y_{2 m}\right)=\Pi\left(x_{2 m+1}\right)$, with $x_{1}=y_{2 m+1}$. That is, $\bigcup_{i=1}^{2 m+1} \Pi\left(C\left(z_{i}\right)\right)$ is an embedded circle of $S(E)$ centered at $\Pi\left(F_{\sigma}\right)$.

This implies that $\Pi\left(\bigcup_{i+1}^{2 m} D\left(z_{i}\right)\right)$ is homeomorphic to a disc. We claim that there exists a topological embedding

$$
H: V=\left\{p \in \mathbb{R}^{2}:|p| \leq \varepsilon\right\} \rightarrow S(E)
$$


such that $H(0)=\Pi\left(z_{1}\right)$ and $\left.H\right|_{V \backslash\{0\}}$ is smooth. This map $H$ will be the parametrization needed to prove that $S(E)$ can be provided with a smooth structure around the multi-saddle point $\Pi\left(F_{\sigma}\right)$. Now we shall prove the claim.

It will be convenient to use complex notation for the elements of $\mathbb{R} \times[-1,1]$ and $\mathbb{R}^{2}$. Let

$$
V_{k}=\left\{r e^{i \theta}: 0 \leq r \leq \varepsilon ; \frac{(k-1)}{m} \pi \leq \theta \leq \frac{k}{m} \pi\right\}
$$

for $k=1,2, \ldots, 2 m$. Let $H_{k}: V_{k} \longrightarrow D\left(z_{k}\right)$ be given by $H_{k}\left(r e^{i \theta}\right)=z_{k}+r e^{i(\theta m+\pi)}$. The sequence of maps $H_{1}, H_{2}, \ldots, H_{2 m}$ induces a map

$$
H: V \longmapsto \Pi\left(\bigcup_{k=1}^{2 m} D\left(z_{k}\right)\right)
$$

where $V=\bigcup_{k=1}^{2 m} V_{k}=\left\{r e^{i \theta}: 0 \leq r \leq \varepsilon\right\}$ such that if $p \in V_{k}$, for some $k$, then $H(p)=\Pi\left(H_{k}(p)\right)$. It is easy to see that $H$ is a well-defined homeomorphism and that $\left.H\right|_{V-\{0\}}$ is smooth. This proves that $S(E)$ has a smooth structure around $\Pi\left(F_{\sigma}\right)$. As $\sigma \in\{1,2, \ldots, \ell\}$ is arbitrary, it follows that $S(E)$ has a smooth structure. This finishes the proof of (a).

We proceed to prove (b). Take a smooth unitary vector field $Y$ tangent to $\mathcal{F}$ and defined in $S(E) \backslash \bigcup_{i=1}^{\ell} \Pi\left(F_{i}\right)$.

Using the methods of [4, page 41] we can find a smooth function $\varphi: S(E) \longmapsto$ $[0,1]$ such that $X=\varphi Y$ extends to a smooth vector field on $S(E)$ whose singularities are exactly $\Pi\left(F_{1}\right), \Pi\left(F_{2}\right), \ldots, \Pi\left(F_{\ell}\right)$. The remaining claims in (b) are immediate. This completes the proof of (b) and of this lemma.

The construction described in the proof of Lemma 8 is close to the known "zippered rectangles" technique introduced by W. Veech in order to prove Keane's conjecture that almost every interval exchange is uniquely ergodic (see [12]).

\section{Structure theOrem AND QUASI-Minimal RECURRENCE REGIONS}

The Structure Theorem of [4] will be used to obtain, in the case of flows without wandering points, the stronger version presented in Theorem 12 below which describes the behavior of such flows around their nontrivial recurrent trajectories.

Lemma 9. Let $\varphi: \mathbb{R} \times M \rightarrow M$ be a continuous flow, without wandering points, on a compact $C^{\infty} 2$-manifold $M$. Let $\gamma$ be a nontrivial recurrent trajectory of $\varphi$. There exists a circle $\mathcal{C}$ passing through $\gamma$ which is transverse to $\varphi$, such that the Poincaré first return map $f: \mathcal{C} \rightarrow \mathcal{C}$, induced by $\varphi$, is conjugate to some minimal $C E T, E: \mathbb{S}^{1} \rightarrow \mathbb{S}^{1}$ and, for some homeomorphism $h: \mathcal{C} \rightarrow \mathbb{S}^{1}$, the diagram

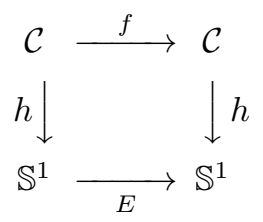

commutes.

Proof. There exists a circle $\mathcal{C}$ passing through $\gamma$ which is transverse to $\varphi$ (see [11, [2. Lemma 2 of 1, p.312]). The Poincaré first return map $f: \mathcal{C} \rightarrow \mathcal{C}$ induced by $\varphi$ 
is semi-conjugate to some CET $E: \mathbb{S}^{1} \rightarrow \mathbb{S}^{1}$ (see [3, Lemma 5], 6]) such that for some surjective and continuous map $h: \mathcal{C} \rightarrow \mathbb{S}^{1}$ of degree one, the diagram of the statement of the lemma commutes.

While $f$ and $E$ are not necessarily defined everywhere, $h$ is defined in the whole $\mathcal{C}$. Under the current assumptions, the map $h$ is a homeomorphism; in fact, given $x$ in the domain of definition $\mathcal{D}(f)$ of $f$, the set $h^{-1}(x)$ is either a point or an interval, but the second case cannot happen because $\varphi$ has no wandering points. It follows that $h$ is a conjugacy between $f$ and $E$. Since $\mathcal{C}$ can be constructed in such a way that it does not intersect the (compact) set of multi-saddle points and of multi-saddle connections of the flow, it follows from Lemma 7 that both $E$ and $f$ are minimal.

Lemma 10. Suppose that we are under the assumptions of Lemma 9, If $p \in \bar{\gamma}$ but the positive (resp. negative) half-trajectory $\gamma_{p}^{+}$(resp. $\left.\gamma_{p}^{-}\right)$passing through $p$ does not meet $\mathcal{C}$, then $\omega\left(\gamma_{p}^{+}\right)$(resp. $\alpha\left(\gamma_{p}^{-}\right)$) is a multi-saddle point.

Proof. The proof is similar to that given in [11, [10, pages 144-146], for the case of vector fields with hyperbolic singularities.

Using the notation introduced in the statement of Lemma 9 and its proof we denote $\mathcal{N}(f)=\mathcal{C} \backslash \mathcal{D}(f)$. For $q \in \mathcal{D}(f)$, we denote by $0<r_{+}(q)<\infty$ the (forward) first return time to $\mathcal{C}$, that is,

$$
f(q)=\varphi\left(q, r_{+}(q)\right)
$$

In the same way if $q \in \mathcal{D}\left(f^{-1}\right)$ we denote by $\infty<r_{-}(q)<0$ the (backward) first return time to $\mathcal{C}$, i.e.,

$$
f^{-1}(q)=\varphi\left(q, r_{-}(q)\right)
$$

Observe that if $q \in \mathcal{D}\left(f^{-1}\right)$, then $f^{-1}(q) \in \mathcal{D}(f)$ and $r_{-}(q)=-r_{+}\left(f^{-1}(q)\right)$. If $q \in \mathcal{N}(f)$ it follows from Lemma 10, that the positive half-trajectory starting at $q$ is a separatrix of some multi-saddle point of the flow $\varphi$. The same is true for a negative half-trajectory starting at a point of $\mathcal{N}\left(f^{-1}\right)$. It follows that $r_{+}(q) \rightarrow \infty$ (resp. $\left.r_{-}(q) \rightarrow-\infty\right)$ when $q$ approaches points of $\mathcal{N}(f)\left(\right.$ resp. $\left.\mathcal{N}\left(f^{-1}\right)\right)$. We define $r_{+}(q)=\infty$ for $q \in \mathcal{N}(f)$ and $r_{-}(q)=-\infty$ for $q \in \mathcal{N}\left(f^{-1}\right)$. Consider the sets

$$
\begin{aligned}
& \mathcal{V}_{+}:=\left\{\varphi(t, q): q \in \mathcal{C}, 0 \leq t \leq \frac{r_{+}(q)}{2}\right\} \text { and } \\
& \mathcal{V}_{-}:=\left\{\varphi(t, q): q \in \mathcal{C}, \frac{r_{-}(q)}{2} \leq t \leq 0\right\}
\end{aligned}
$$

see Figure 4.

Lemma 11. Let $\mathcal{V}=\mathcal{V}_{-} \cup \mathcal{V}_{+}$. There exists a homeomorphism $H: \mathcal{V} \rightarrow S(E)$ which is a topological equivalence between $(\varphi, \mathcal{V})$ and $(\Phi, S(E))$. Moreover $\overline{\mathcal{V}}$ is a quasi-minimal recurrence region.

Proof. Let $\left(f_{\lambda}\right)$ with $\lambda \geq 1$ be a continuous family of homeomorphisms

$$
f_{\lambda}:[-\lambda, \lambda] \rightarrow[-1,1]
$$

which converges uniformly in the compact parts of $(-\infty, \infty)$ to a homeomorphism $f_{\infty}:(-\infty, \infty) \rightarrow(-1,1)$. We will demand that $f_{\lambda}(0)=0$ and $\left(t \cdot f_{\lambda}(t)\right)>0$ for all $t$ and $1 \leq \lambda \leq \infty$ 
For all $q \in \mathcal{C}$ and $r_{-}(q) / 2 \leq t \leq r_{+}(q) / 2$, define the number

$$
\lambda(t, q)= \begin{cases}\frac{r_{+}(q)}{2} & \text { if } t \geq 0, \\ \frac{r_{-}(q)}{2} & \text { if } t<0 .\end{cases}
$$

Now if $\varphi(t, q)$ is a point in $\mathcal{V}$ with $r_{-}(q) / 2 \leq t \leq r_{+}(q) / 2$, we define $\tilde{H}: \mathcal{V} \rightarrow$ $\mathbb{S}^{1} \times[-1,1]$ as $\tilde{H}(\varphi(t, q))=\left(h(q), f_{\lambda(t, q)}(t)\right)$.

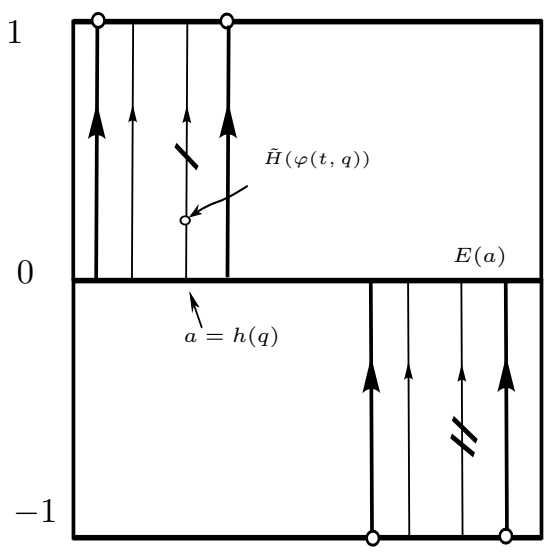

(a)

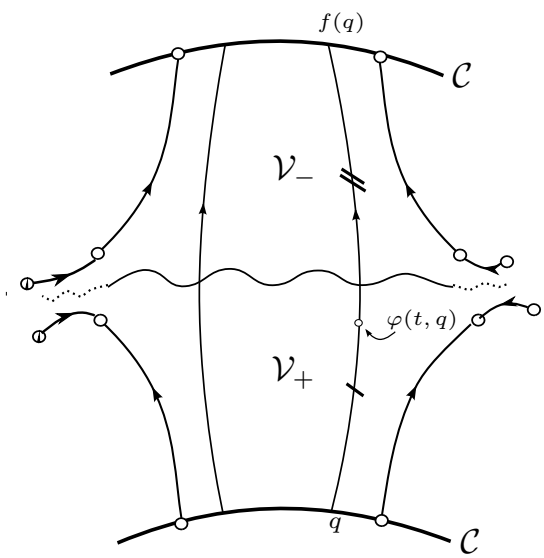

(b)

Figure 4. The homeomorphism $\tilde{H}$.

Define the vertical foliation of $\mathbb{S}^{1} \times[0,1]$ as the one induced by the constant vector field $(0,1)$. It is easy to check that $\tilde{H}$ is a homeomorphism which takes the foliation induced by $\varphi$ in $\mathcal{V}$ onto the vertical foliation of $\mathbb{S}^{1} \times[0,1]$. In fact the following statements hold.

(i) If $q \in \mathcal{D}(f)$, then $\tilde{H}\left(\left\{\varphi(t, q), 0 \leq t \leq r_{+}(q)\right\}\right)=\{(h(q), s), 0 \leq s \leq 1\} \cup$ $\{(E(h(q)), s),-1 \leq s \leq 0\}$.

(ii) If $q \in \mathcal{N}(f)$, then $\tilde{H}\{\varphi(t, q), 0 \leq t<\infty\}=\{(h(q), s), 0 \leq s<1\}$.

(iii) If $q \in \mathcal{N}\left(f^{-1}\right)$, then $\tilde{H}\{\varphi(t, q),-\infty<t \leq 0\}=\{(h(q), s),-1<s \leq 0\}$.

Let $\Pi: \mathbb{S}^{1} \times[-1,1] \rightarrow S(E)$ be the canonical projection. Observe that if $q \in$ $\mathcal{N}(f), \Pi(\{(h(q), s), 0 \leq s<1\})$ is contained in the stable separatrix of a multisaddle point of $\Phi$ passing through $\Pi(h(q))$. In the same way, if $q \in \mathcal{N}\left(f^{-1}\right)$, then $\Pi(\{(h(q), s),-1<s \leq 0\})$ is contained in the unstable separatrix of a multi-saddle point of $\Phi$ passing through $\Pi(h(q))$.

We define $H: \mathcal{V} \rightarrow S(E)$ as $H(\varphi(t, q))=\Pi \circ \tilde{H}(\varphi(t, q))$ for $\varphi(t, q) \in \mathcal{V}$ with $q \in \mathcal{C}$ and $r_{-}(q) / 2 \leq t \leq r_{+}(q) / 2$. It can be seen that $H$ is a well-defined homeomorphism that performs the required topological equivalence. Certainly $\mathcal{V}$ is a quasi-minimal set. On the other hand, by Lemma 10, the boundary of $\mathcal{V}$ is made up of multisaddle points and multi-saddle connections; this implies the last statement of the lemma. 
Theorem 12. Let $\varphi: \mathbb{R} \times M \rightarrow M$ be a continuous flow, without wandering points, on a compact $C^{\infty} 2$-manifold $M$. Then the closures of the nontrivial recurrent trajectories of $\varphi$ determine finitely many quasi-minimal recurrence regions $\Omega_{1}, \Omega_{2}, \ldots, \Omega_{m}$ of $\varphi$ such that, given $i, j \in\{1,2, \ldots, m\}$, the following conditions hold.

(a) If $i \neq j, \Omega_{i} \cap \Omega_{j} \subset \partial \Omega_{i} \cup \partial \Omega_{j}$.

(b) There exists a circle $\mathcal{C}_{i} \subset \Omega_{i}$ transverse to $\varphi$ such that the forward Poincaré map $f_{i}: \mathcal{C}_{i} \rightarrow \mathcal{C}_{i}$ (induced by $\varphi$ ) is topologically conjugate to a minimal interval exchange map $E_{i}: \mathbb{S}^{1} \rightarrow \mathbb{S}^{1}$.

(c) Let $V_{i}$ denote the complement in $\Omega_{i}$ of the set of singularities and saddle connections of $\varphi$. Let $\left(\phi_{i}, S\left(E_{i}\right)\right)$ be a suspension of $E_{i}$ and let $F_{i}$ be the set of singularities of $\phi_{i}$. Then the restriction of $\phi_{i}$ to $S\left(E_{i}\right) \backslash F_{i}$ is topologically equivalent to the restriction of $\varphi$ to $V_{i}$.

Proof. It follows from Lemma 11 that the closure of a nontrivial recurrent trajectory determines a quasi-minimal recurrence region which contains a circle transverse to the flow. There can only be finitely many quasi-minimal recurrence regions because the circles (transverse to the flow) that these regions contain cannot be either nullhomotopic or homotopic to each other. The remaining parts of this theorem follow from the previous Lemmas 8,9 and 11 .

Proof of Theorem 3. Let $\gamma$ be a two-sided periodic trajectory. Then $\gamma$ has open neighborhoods which are cylinders filled out by two-sided closed orbits. Let $\mathcal{V}$ be the union of all these cylindrical neighborhoods. We claim that $\overline{\mathcal{V}}$ is a semicylindric region. In fact, by the structure of the quasi-minimal recurrence regions, $\mathcal{V}$ is disjoint from the interior of any recurrence region. Therefore, by Corollary 6 , the boundary $\partial \mathcal{V}$ of $\mathcal{V}$, when not empty, can only contain singularities, one-sided closed orbits and graphs. There are several cases to be considered. Notice that cases (a)-(d) below have already been considered (see Remark 1 and the preceding commentaries).

(a) If $\partial \mathcal{V}$ consists of two center points $p$ and $q$, then $M=\mathcal{V} \cup\{p\} \cup\{q\}$ is the 2-sphere.

(b) If $\partial \mathcal{V}$ consists of a center point $p$ and a one-sided closed orbit $\gamma$, then $M=\mathcal{V} \cup\{p\} \cup \gamma$ is the 2 -projective space.

(c) If $\partial \mathcal{V}$ consists of two one-sided closed orbits $\gamma, \sigma$, then $M=\mathcal{V} \cup \sigma \cup \gamma$ is the Klein bottle.

(d) If $\partial \mathcal{V}$ is empty, then $\mathcal{V}=\overline{\mathcal{V}}$ is the 2-torus.

(e) $\partial \mathcal{V}$ does not consist of a unique one-sided closed curve; otherwise, $\left.\varphi\right|_{\overline{\mathcal{V}}}$ would induce a non-orientable foliation, which would be a contradiction.

(f) $\partial \mathcal{V}$ cannot consist of exactly one center; otherwise, $\overline{\mathcal{V}}$ would an orientable manifold of Euler characteristic one (i.e., the projective plane), which is not possible.

Therefore, if $\partial \mathcal{V}$ is connected it must be a graph of $\varphi$. In conclusion, $\overline{\mathcal{V}}$ is a semicylindric region.

It is easy to see that one-sided closed curves are isolated. Therefore, $\varphi$ can only have finitely many one-sided closed curves, singularities and multi-saddle connections. Again by Corollary [6, it follows that $M$ is the union of finitely many quasi-minimal recurrence regions and semi-cylindric regions. 


\section{ACKNOWLEDGMENTS}

The second author has been partially supported by CAPES-MECD Grants \#071/04 and \#HBP2003-0017, and by CNPq Grants \#470957/2006-9 and \#306328/2006-2, Brazil. The third author was partially supported by a MCYT/ FEDER grant number MTM2008-03437 and by a CIRIT grant number 2009SGR 410.

\section{REFERENCES}

[1] A.A. Andronov, E.A. Leontovich, I.I. Gordon and A.G. Maier, Qualitative Theory of Second Order Dynamical Systems, translated by John Wiley \& Sons, New York, 1973. MR0350126 (50:2619)

[2] C. Gutierrez, Structural stability for flows on the torus with a cross-cap, Trans. Amer. Math. Soc. 241 (1978), 311-320. MR 492303 (80k:58065)

[3] C. Gutierrez, Smoothability of Cherry flows on two-manifolds, in Geometric Dynamics, Springer Lecture Notes in Mathematics 1007 (1981), 308-331. MR730275 (85h:58148)

[4] C. Gutierrez, Smoothing continuous flows on two-manifolds and recurrences, Ergod. Th. \& Dynam. Sys. 6 (1986), 17-44. MR837974 (87k:58222)

[5] P. Hartman, Ordinary Differential Equations, John Wiley and Sons. Inc., 1964. MR0171038 $(30: 1270)$

[6] A. Katok And B. Hasselblatt, Introduction to the modern theory of dynamical systems, with a supplementary chapter by Katok and Leonardo Mendoza, Encyclopedia of Math. and its Appl. 54, Cambridge University Press, 1995. MR.1326374 (96c:58055)

[7] M. Keane, Interval Exchange Transformations, Math. Z. 141 (1975), 25-31. MR0357739 $(50: 10207)$

[8] N. Markley, The Poincaré-Bendixson theorem for the Klein bottle, Trans. Amer. Math. Soc. 135 (1969), 159-165. MR0234442 (38:2759)

[9] I. Nikolaev and E. Zhuzhoma, Flows on 2-dimensional manifolds, Springer Lecture Notes in Mathematics, Vol. 1705, Berlin, 1999. MR1707298 (2001b:37065)

[10] J. Palis, JR. And W. DE Melo, Geometric Theory of Dynamical Systems, Springer-Verlag, New York, Heidelberg Berlin, 1982. MR.0669541 (84a:58004)

[11] M. Peiхото, Structural stability on two-dimensional manifolds, Topology 1 (1962), 101-120. MR0142859 (26:426)

[12] W. A. VEECH, Gauss measures for transformations on the space of interval exchange maps, Ann. of Math. (2) 115 (1982), 201-242. MR644019 (83g:28036b)

Departamento de Matemática, Universidade Federal do Espírito Santo, Av. FerNANDO FERRARI 514, Vitoria, ES 19075-910 BRAZIL

E-mail address: milton.e.cobo@gmail.com

Departamento de Mateática, Instituto de Ciências Matemáticas e de Computação, Universidade de Sao Paulo, CxP 668, São Carlos, SP, 13560-970 Brazil

Departament de Matemàtiques, Universitat Autònoma de Barcelona, 08193 Bellaterra, Barcelona, Catalonia, Spain

E-mail address: jllibre@mat.uab.cat 